\title{
Strong and tough graphene papers constructed with pyrene-containing small molecules via $\pi-\pi / H-$ bonding synergistic interactions
}

\author{
Hong Yuan ${ }^{1}$, Liangbing $\mathrm{Ge}^{1}$, Kun $\mathrm{Ni}^{1}$, Xiukai Kan ${ }^{3}$, Si-Ming Chen ${ }^{2}$, Mengting Gao ${ }^{3}$, Fei Pan ${ }^{1}$, \\ Jianglin $\mathrm{Ye}^{1}$, Fang $\mathrm{Xu}^{1}$, $\mathrm{Na} \mathrm{Shu}{ }^{1}$, Jieyun $\mathrm{Li}^{1}$, Tao Suo ${ }^{3}$, Shu-Hong $\mathrm{Yu}^{2}$ and $\mathrm{Yanwu} \mathrm{Zhu}^{1 *}$
}

\begin{abstract}
Lightweight yet strong paper with high toughness is desirable especially for impact protection. Herein we demonstrated electrically conductive and mechanically robust paper (AP/PB-GP) made of reduced graphene oxide via interfacial crosslinking with 1-aminopyrene (AP) and 1-pyrenebutyrat (PB) small molecules. The AP/PB-GP with thickness of over ten micrometer delivers a record-high toughness $\left(\sim 69.67 \pm 15.3 \mathrm{MJ} \mathrm{m}^{-3}\right.$ in average), simultaneously with superior strength (close to $1 \mathrm{GPa}$ ), allowing an impressive specific penetration energy absorption $\left(\sim 0.17 \mathrm{MJ} \mathrm{kg}^{-1}\right)$ at high impact velocities when used for ballistic impact protection. Detailed interfacial and structural analysis reveals that the reinforcement is synergistically determined by $\pi-\pi$ interaction and $\mathrm{H}$-bonding linkage between adjacent graphene lamellae. Especially, the defective pores within the graphene platelets benefit the favorable adsorption of the pyrene-containing molecules, which imperatively maximizes the interfacial binding, facilitating deflecting crack and plastic deformation under loading. Density functional theory simulation suggests that the coupling between the polar functional groups, e.g., $-\mathrm{COOH}$, at the edges of graphene platelets and $-\mathrm{NH}_{2}$ and $-\mathrm{COOH}$ of $\mathrm{AP} / \mathrm{PB}$ are critical to the formation of hydrogen bonding network.
\end{abstract}

Keywords: graphene paper, $\pi-\pi$ interaction, H-bonding, synergistic reinforcement, mechanical properties

\section{INTRODUCTION}

Due to the strong $\mathrm{sp}^{2}$ conjugation, graphene theoretically owns a Young's modulus of $1 \mathrm{TPa}$, a tensile strength of $130 \mathrm{GPa}$ and an electrical conductivity of $10^{6} \mathrm{~S} \mathrm{~cm}^{-1}$, supplementing mechanical and electrical merits in numerous graphene-based applications [1-3]. The graphitic domains provide abundant sites for $\pi-\pi$ interaction; assembling graphene platelets into well-defined architectures has been an efficient way to realize the superior performances on the macroscale [4-6]. As representative assemblies, free-standing yet flexible graphene paper (abbreviated as GP), has recently earned a great deal of attention, for its potential applications in energy storage, protective layers, chemical filters, mechanical sensors, and heat dissipation [7-9]. In many applications, the GP with simultaneously superior strength and toughness is especially valuable as such paper would provide light yet strong protection while with the ability to absorb impact. However, bare GP, e.g., reduced graphene oxide (RGO) paper fabricated by stacking GO platelets via layer-bylayer assembly, vacuum filtration or tape casting, followed by reduction, demonstrated unsatisfactory mechanical performance due to factors such as structural defects, inhomogeneous lateral alignment or deficiency in the interface robustness $[5,10,11]$. To further improve the mechanical performance of GP, one way is to optimize the components of the building blocks by incorporation of various inorganic fillers such as carbon nanotubes, nanofibers or transition metal sulfide nanosheets $[12,13]$. On the other hand, it is critical to improve the alignment of lamella and the interfacial interactions for strong and

\footnotetext{
${ }^{1}$ Hefei National Laboratory for Physical Sciences at the Microscale \& CAS Key Laboratory of Materials for Energy Conversion, Department of Materials Science and Engineering, iChEM, University of Science and Technology of China, Hefei 230026, China

${ }^{2}$ Division of Nanomaterials and Chemistry, Hefei National Laboratory for Physical Sciences at the Microscale, CAS Center for Excellence in Nanoscience, Hefei Science Center of CAS, Collaborative Innovation Center of Suzhou Nano Science and Technology, Department of Chemistry, University of Science and Technology of China, Hefei 230026, China

${ }^{3}$ School of Aeronautics, Northwestern Polytechnical University, Xi'an 710072, China

* Correspondence author (email: zhuyanwu@ustc.edu.cn)
} 
tough GP $[3,14]$. For instance, a plasticization stretching method has been used to improve the alignment of graphene lamella for an ultra-high tensile strength of $\sim 1.1 \mathrm{GPa}[14]$ but at the cost of toughness (elongation of $\sim 1.6 \%$ ). In this sense, design principles can be learned from natural nacre, in which the 'brick and mortar' architecture, embraced with $\sim 95$ vol.\% aragonite calcium carbonate platelets and $\sim 5$ vol. $\%$ proteinaceous adhesive binders, ensures the remarkable strength and toughness as the abundant inorganic-organic interfaces endure large inelastic deformations and dissipate much energy during fracturing $[15,16]$.

Numerous strategies have been inspired for the fabrication of nacre-like hybrid GP, with mechanical performances surpassing those of bare GP or even natural nacre [17-19]. In such pursuit, the interfacial interactions have been achieved by hydrogen $(\mathrm{H})$-bonding, ionic bonding and $\pi-\pi$ interaction, bridging adjacent graphene platelets with organic binders such as cellulose, chitosan (CS) and other adhesive biopolymers [10,20,21]. Through synergistic ionic/H-bonding strengthening, for example, the hybrid GP crosslinked by $\mathrm{Al}^{3+}$ and carboxymethyl cellulose (CMC) delivered a tensile strength of up to $586.6 \pm$ $12 \mathrm{MPa}$ and a toughness of up to $12.1 \pm 0.44 \mathrm{MJ} \mathrm{m}^{-3}$ [22]. By the strong interaction between RGO and $\mathrm{Cu}^{2+}$ ligandcoordinated CS molecular chains, the $3-\mu \mathrm{m}$-thick RGO/ CS-Cu paper has demonstrated significantly enhanced tensile strength of $868.6 \pm 40.6 \mathrm{MPa}$ with a toughness of $14.1 \pm 1.20 \mathrm{MJ} \mathrm{m}^{-3}$ [23]. In spite of the greatly improved interfacial strength, the strong GP mentioned above has been obtained at the cost of toughness and ductility. This is partially due to the fact that it is challenging to uniformly distribute the stiff polysaccharide biopolymers with branched chains (as typically used above) between graphene platelets, readily resulting in a concentration of stress and failure under small strains [24,25]. In contrast, the incorporation of flexible polymers, e.g., poly(vinyl alcohol) (PVA), polydopamine (PDA) or polyurethane (PU) into graphene lamellae, may contribute to higher toughness through accommodating more deformation, whereas it frequently leads to restricted strength [26-28]. For example, a highly stretchable GO/PVA composite paper (with a break elongation of up to $\sim 195 \%$ ) delivered ultra-high toughness but limited strength $(\sim 115 \mathrm{MPa})$ [27].

To realize simultaneously high tensile strength and toughness, recent efforts have utilized pyrene-containing linear molecules (e.g., 1-pyrenebutyric acid $N$-hydroxy succinimide ester 1-aminopyrene (PSE-AP) [29], 1-aminopyrene (AP)-disuccinimidyl suberate (AD) [30], bis(1- pyrene methyl) docosa-10,12-diynedioate (BPDD) [31]) to optimize the alignment of graphene platelets and the interfacial strength via the formation of $\pi-\pi$ conjugation (from pyrene) and $\mathrm{H}$-bonding linkage (from linear chains) [26,30-33]. For example, 5- $\mu \mathrm{m}$-thick MXenefunctionalized and $\mathrm{AD}$-conjugated molecule-crosslinked GP provided a strength of $\sim 699.1 \mathrm{MPa}$ and high toughness of $\sim 42.7 \mathrm{MJ} \mathrm{m}^{-3}$ [30]. By optimizing the pyreneterminal long-chain molecule via esterification reaction of 1-pyrene methanol with docosa-10,12-diynedioic acid (DDA), 1-ethyl-3-(3-dimethylaminopropyl) carbodiimide hydrochloride (EDCI) and 4-dimethylaminopyridine (DMAP), the resultant 3.4- $\mu \mathrm{m}$-thick, BPDD-decorated GP delivered a strength of up to $1054.2 \pm 56.5 \mathrm{MPa}$, accompanying an impressive toughness of $35.8 \pm 1.9 \mathrm{MJ} \mathrm{m}^{-3}$ [31]. Recently, 4- $\mu$ m-thick hybrid graphene films (RGO/ $\mathrm{BP}-\mathrm{AD}$ ) functionalized with black phosphorus (BP)/pyrene-containing linear molecules have shown a record high toughness of $\sim 51.8 \mathrm{MJ} \mathrm{m}^{-3}$ by combining covalent bonding P-O-C between GO and BP nanosheets and $\pi-\pi$ stacking interactions by the long-chain molecule $\mathrm{AD}$ [33]. Indeed, the $\pi-\pi$ bridging long-chain linkages not only bond neighboring graphene platelets but also provide space for the movement of platelets to transfer local stress. But the syntheses of long-chain molecules like PSE, BPDD and [DSS] [AD] are relatively complicated; the thicknesses of these functionalized graphene films are limited to several micrometers and the controllability is challenging in scalable preparations. In addition, it has been proved that multilayer graphene (10-100 nm thick) is an extraordinary armor material exhibiting excellent impact energy upon a supersonic penetration [34]. Therefore, the thickness of GP needs to be scaled up towards practical applications and the interface in thick paper shall be specifically designed to bear impact more efficiently.

We have noticed that the 1-pyrenebutyrate $(\mathrm{PB})$ as a stabilizer and conductive additive can facilitate charge transfer between aromatic molecules and RGO sheets by strong affinity via $\pi-\pi$ stacking and its side alkyl chain can form cohesive network to increase flexibility [35]. Although the conjugated AP molecules, being highly aromatic in nature, is known to interact strongly with the basal plane of graphene sheets via $\pi-\pi$ stacking, the intricate interplay mechanisms need to be further elucidated. Herein, we report a straightforward immersion strategy to reinforce the graphene platelets with such two pyrene-containing molecules. The experimental results indicate that the two conjugated monomers combined together also show amazing reinforcing effects on the 
strength and toughness of GP without complicated esterification reaction process. With an optimal $\mathrm{AP} / \mathrm{PB}$ concentration ratio of $\sim 4: 1$, the obtained thick (raised to 10-15 $\mu \mathrm{m})$ AP/PB-GP demonstrates a record-high toughness $\left(69.67 \pm 15.3 \mathrm{MJ} \mathrm{m}^{-3}\right)$ while maintaining the superior strength $(974 \pm 98 \mathrm{MPa})$. Due to the strong and tough structural feature, the thicker AP/PB-GP (thickness: $25-80 \mu \mathrm{m}$ ) also shows an impressive specific penetration energy $\left(\sim 0.17 \mathrm{MJ} \mathrm{kg}{ }^{-1}\right.$, at $\left.V_{0} \approx 313 \mathrm{~m} \mathrm{~s}^{-1}\right)$, outperforming steel, ductile aluminum, and amorphous glassy polymer [34,36], and improves the merits especially in impact resistance application. The detailed structural analysis by in-situ X-ray diffraction (XRD) under external uniaxial stress shows larger interlayer distance with broader distribution. The surface force mapping of adhesion, performed with AP-modified atomic force microscopy (AFM) on graphene platelets, indicates that the pores in graphene platelets enhance the adhesion to AP molecules. The density functional theory (DFT) simulation further suggests that the reinforcement is determined by the synergistic interactions of $\pi-\pi$ stacking and $\mathrm{H}$-bonding linkages in adjacent graphene lamellae, among which the latter is formed between the amine or carboxyl groups in AP/PB and polar functional groups (e.g., $-\mathrm{COOH}$ ) at the edges of graphene platelets.

\section{EXPERIMENTAL SECTION}

\section{Chemicals and materials}

Graphite powder (325 mesh, $\sim 45 \mu \mathrm{m}$ ) was used as received from Alfa Aesar. AP and PB were purchased from Sigma-Aldrich. $N, N^{\prime}$-dimethylformamide (DMF) was obtained from Tianjin Chemical Reagent Company. Other analytical grade reagents, e.g., potassium permanganate $\left(\mathrm{KMnO}_{4}\right)$, sulphuric acid $\left(\mathrm{H}_{2} \mathrm{SO}_{4}, 98\right.$ wt.\%), hydrogen peroxide $\left(\mathrm{H}_{2} \mathrm{O}_{2}\right)$, hydroiodic acid (HI, 55 wt.\%) and ethanol obtained from Sinopharm Chemical Reagent Co., Ltd., were used without further purification. Ultrapure doubly deionized water (resistivity $>18.2 \mathrm{M} \Omega$ ) was obtained from a synergy UV water purification system (Millipore Corporation, Direct-Q3, US). Mixed cellulose ester (MCE) membrane filter (Xinya Purification, Co. Ltd. China) with $0.45 \mu \mathrm{m}$ pore size and $47 \mathrm{~mm}$ diameter was used in filtration to support the fabricated paper. Sonication was performed using a KQ-300VDE dual frequency ultrasonic cleaner from Ultrasonic Instruments (KQ-700TDE, Shumei Co. Ltd, China). The commercial GO paper (thickness: $\sim 60$ or $80 \mu \mathrm{m}$ ) was obtained from Changzhou Fuxi Technology Co. Ltd, China.

\section{Fabrication of GOP and GP}

Typically, the colloidal GO dispersions were synthesized by milder oxidation at low temperature [37]. GO papers (GOPs) were produced by the assembly of the aforementioned colloidal GO dispersions $\left(\sim 2 \mathrm{mg} \mathrm{mL}^{-1}\right)$ with vacuum filtration through a cellulose ester membrane $(0.45 \mu \mathrm{m}$ pore size). The thickness $(10-25 \mu \mathrm{m})$ of the paper was adjusted by the volume and concentration of GO dispersion. After repeated filtration, the dried GO specimens could fall off spontaneously from the filter membrane to form freestanding laminated GOP, followed by reduction in $\mathrm{HI}$ solution ( $55 \mathrm{wt} . \%$ ) for $24 \mathrm{~h}$ in the atmosphere. Subsequently, the resultant silver-gray GP was repeatedly immersed by ethanol to remove iodine ions till the solution retained transparent and then was dried for subsequent use.

\section{Fabrication of AP-GP, PB-GP, AP/PB-GP}

Before the fabrication of AP/PB-GP, the PB-GP was initially obtained through immersing the GP in $5 \mathrm{mmol} \mathrm{L}^{-1}$ $\mathrm{PB} / \mathrm{DMF}$ solution for $6 \mathrm{~h}$ to realize preferential interlayer adsorption [35]. Subsequently, the resultant PB-GP was soaked in $20 \mathrm{mmol} \mathrm{L}^{-1}$ AP/DMF solution for $24 \mathrm{~h}$ and rinsed thoroughly with ethanol to remove residual DMF solvent, then dried at ambient condition to obtain AP/ PB-GP. It is difficult to accurately determine the content of conjugated molecules in the resultant paper due to the undefined adsorption content. Therefore, the optimized adsorption ratios were determined through soaking $\mathrm{PB}$ cross-linked GP in the certain AP/DMF solution for long time $(\sim 24 \mathrm{~h})$ to make sure an efficient surface adsorption and anchoring of small molecules. For details, the samples with different immersion concentration ratios (AP/ PB of 1:1, 2:1, 3:1, 4:1, 5:1, 6:1, 7:1) were prepared by immersing PB-GP into AP/DMF solutions with different concentrations of AP $(5,10,15,20,25,30$ and $35 \mathrm{mmol} \mathrm{L}^{-1}$ ) to adsorb AP molecules for another $24 \mathrm{~h}$, followed by rinsing with ethanol and drying at ambient condition. The AP-DMF/PB-DMF solutions could be easily recycled and reused for several times. The optimized single cross-linked AP-GP samples were fabricated through soaking bare GP in a $20 \mathrm{mmol} \mathrm{L}^{-1} \mathrm{AP} / \mathrm{DMF}$ solution for $24 \mathrm{~h}$ without $\mathrm{PB}$ immersion. All the hybrid papers were rinsed thoroughly with ethanol to remove the residual solvents and dried at ambient condition for use.

\section{Fabrication of TGP, AP/PB-TGP, CGP, AP/PB-CGP}

To further show the scalability of preparation, the GOPs were also made by the tap casting process (abbreviated as TGOP). In a typical process, GO slurry $\left(\sim 6 \mathrm{mg} \mathrm{mL}^{-1}\right.$, 
$50 \mathrm{~mL}$ ) was poured onto a glass plate, and then dried in a vacuum oven at $35^{\circ} \mathrm{C}$ for $48 \mathrm{~h}$ to evaporate the solvent. Subsequently, the dried TGOP could fall off spontaneously from the glass to form freestanding laminated paper. The thickness of TGOP was controlled as $\sim 18 \mu \mathrm{m}$. In addition, the commercial GOP (abbreviated as CGOP, with thickness of $\sim 60$ or $80 \mu \mathrm{m}$ ) made by blade coating process was directly obtained from Changzhou Fuxi Technology Co. Ltd, China. In a typical process, the blade coating spreads the GO slurry over planar substrate to get continuous solid paper after drying. The reduction of TGOP and CGOP was the same as in the HI solution (55 wt.\%) in the atmosphere for $24 \mathrm{~h}$ to obtain the TGP and CGP, and then the crosslinking treatment was performed under the optimal $\mathrm{AP} / \mathrm{PB}$ concentration ratio of $4: 1$ to obtain the resultant AP/PB-CGP and AP/PB-TGP.

\section{Characterizations}

Scanning electron microscopy (SEM) images were obtained with field-emission scanning electron microscope (FEI, Apreo, $2 \mathrm{kV}$, US); the typical thickness of the GO platelets was measured by atomic force microscope (AFM, Nanoscope III MultiMode, Germany). High-resolution transmission electron microscopy (HRTEM, JEOL-2100F, Japan) was carried out to characterize the microstructure of AP-decorated graphene sheets. Raman spectra (LabRAM, RM3000, Renishaw, UK, $532 \mathrm{~nm}$ laser) and Fourier transform infrared (FTIR, attenuated total reflection mode, Thermo Nicolet nexus-470, US) spectra were obtained to characterize the microstructure of the hybrid GP. The assembly of the paper was evaluated by XRD with $\mathrm{Cu} K a$ radiation $(\lambda=1.5418 \AA$, tube voltage $40 \mathrm{kV}$, tube current $30 \mathrm{~mA}$, Rigaku SmartLab, Japan). The chemical composition was characterized by X-ray photoelectron spectroscopy (XPS) on Thermo ESCALAB 250 (US) with $\mathrm{Al} \mathrm{Ka}$ radiation $(h v=1486.6 \mathrm{eV})$, and all spectra were calibrated to the binding energy of $\mathrm{C}=\mathrm{C}$ bonds $(284.6 \mathrm{eV})$. The electrical conductivity was measured by a standard four-probe method using a Hall Effect testing system (Keithley 2182A/6430, China).

\section{Mechanical property testing}

The mechanical performance of the paper was evaluated by uniaxial tensile tests performed in ambience with a mechanical testing machine (Instron $5565 \mathrm{~A}$, US). At least three strips from the same paper with similar thickness $(\sim 10 \mu \mathrm{m})$ were measured for the mechanical tensile test. The toughness was calculated by the integral area of the stress-strain curves. The dynamic impact resistance test was conducted at a homemade single stage gas gun measuring system based on specimens with different thicknesses $(25-80 \mu \mathrm{m})$ and the diameter of impact steel ball is $200 \mu \mathrm{m}$. Three points were impacted at different positions for the same paper. Various initial impact velocities (70 to $350 \mathrm{~m} \mathrm{~s}^{-1}$ ) were generated with a gas gun applied along the out-of-plane direction of the paper and high-speed camera (frame rate 110,000 fps, field of vision $304 \times 136 \mathrm{pt}$, pixel distance conversion $1 \mathrm{~m}=7050 \mathrm{pt}$ ) was used to capture the impact velocities. Adhesion force measurement was carried out via AFM in peak force tapping mode (Dimension Fast Scan BRUKER, Germany) in vacuum environment in the glove box to avoid the influence of air humidity. To get rid of adhesion contribution from the solvent, DMF-treated bare tips were used as the control samples.

The penetration energy $\left(E_{\mathrm{p}}\right)$ and specific energy absorption $\left(E_{\mathrm{p}}^{*}\right)$ were calculated with the following equations $[34,36]$ :

$E_{\mathrm{p}}=1 / 2 m\left(V_{0}^{2}-V_{\mathrm{r}}^{2}\right)$,

$E_{\mathrm{p}}^{*}=E_{\mathrm{p}}\left(\rho A_{\mathrm{s}} h\right)^{-1}$,

where $m$ is the mass of the projectile, $\rho$ is the density of the specimen, $h$ is the specimen thickness and $A_{\mathrm{s}}$ is the area of a strike face $\left(A_{\mathrm{s}}=\pi D^{2} / 4\right.$, strike face diameter $D>>h)$.

\section{Computational methods}

All calculations were performed using DFT [38] with a periodic boundary condition as implemented in the Vienna ab-initio Simulation Package. The exchange-correlation potentials were treated by generalizing gradient approximation (GGA) and parametrized by the PerdewBurke-Ernzerhof (PBE) functional within the plane wave augmented (PAW) method [39]. The Grimme's DFT-D3 [40] method was used to describe the long-range van der Waals interaction. The relaxation of all atomic position was performed with the forces smaller than $0.01 \mathrm{eV} \AA^{-1}$ and an energy cutoff of $500 \mathrm{eV}$. The systems considered here were isolated and the sampling of the Brillouin zone was done with equivalent of $1 \times 1 \times 1$ Monkhorst-Pack $k$ point grids for all supercells [41]. For details, two graphene sheets partially decorated by carboxyl and hydroxyl groups were constructed at their edges combining the experimental XPS results; the remaining parts were saturated with hydrogen atoms, and kept parallel with the interlayer distance of $3.54 \AA$. This was equivalent to the experimental XRD result in a box of $23 \times 20 \times 20 \AA^{3}$. We firstly discussed the coupling of the main functional groups (amino, hydroxyl, carboxyl groups). As shown in Table S1, weaker hydrogen bonds exist between AP and 
PB molecules due to the existence of electron donor group at the edge of $\mathrm{PB}$ molecules. However, both of them have strong coupling effects on the edge carboxyl groups of graphene.

Two main stretching mechanisms, interlayer slipping (IS) and edge coupling (EC) were mainly considered between AP molecules and graphene sheets. As shown in Fig. S1, a sandwich-like structure with AP molecules inserted between two graphene sheets was built for the IS. Five fixed carbon atoms (labeled as yellow) in the bottom layer extended the bond length of the other five mobile carbon atoms (labeled as green) in the top layer with a distance of $0.1 \AA$ during the whole stretching process (Fig. S2a). The relaxation of atomic position was performed with the forces smaller than $0.01 \mathrm{eV} \AA^{-1}$ and an energy cutoff of $500 \mathrm{eV} .230$ calculations were performed on the basis of the aforementioned calculated optimization process. Note that the length of the box was increased by $0.1 \AA$ in each step along the $x$ direction to avoid the interaction when the top graphene platelets approached the right boundary of the box. Finally, we extracted the force $f$ in the $x$ direction on the five carbon atoms that were fixed in the top graphene sheets in each calculation and plotted the curve of $F$ versus displacement. Here $F$ in unit of $\mathrm{MPa}$, can be converted according to the formula below:

$F=f / L t$,

where $L$ is the lateral size of graphene sheets as indicated in Fig. S2a, $t$ is the thickness of the systems mentioned above, namely interlayer separation in the light of lit- erature [39]. As indicated in the simulated stretching force versus distance curves, we chose "COOH-COOH" coupling with the largest binding energy as the object for the EC mechanism, then the small molecule AP was placed above the two adjacent graphene sheets and optimized and the same stretching process was carried out as before. Force-displacement response was also obtained according to the formula (2). Here charge is defined as:

$\Delta \rho=\rho_{\mathrm{AP}+\mathrm{GHs}}-\rho_{\mathrm{AP}}-\rho_{\mathrm{GHs}}$,

where $\rho_{\mathrm{AP}+\mathrm{GHs}}$ represents the charge density for the GHs with adsorbed AP, $\rho_{\mathrm{AP}}$ is the charge density of an isolated $\mathrm{AP}$, and $\rho_{\mathrm{GHs}}$ is the charge density of graphene sheets.

\section{RESULTS AND DISCUSSION}

The typical synthesis procedure of AP/PB-GP is illustrated in Fig. 1. In brief, a vacuum-assisted filtration was used to fabricate layered GOP from a homogeneous GO aqueous solution with a concentration of $\sim 2 \mathrm{mg} \mathrm{mL}^{-1}$. After repeated filtration, the dried GO films could fall off spontaneously from the filter membrane to form freestanding laminated GOP. Then the brown colored GOP was immersed in hydroiodic acid ( $\mathrm{HI}$ in $\mathrm{H}_{2} \mathrm{O}, 55$ wt.\%) for $24 \mathrm{~h}$ to partially restore the conjugation based on the nucleophilic substitution reaction [42]. Subsequently, the resultant GP was sequentially soaked in optimized $5 \mathrm{mmol} \mathrm{L}^{-1} \mathrm{~PB} / \mathrm{DMF}$ solution for $6 \mathrm{~h}$ and $20 \mathrm{mmol} \mathrm{L}^{-1}$ AP/DMF solution for $24 \mathrm{~h}$, to obtain AP/PB-GP. GP was also separately immersed in $5 \mathrm{mmol} \mathrm{L}^{-1} \mathrm{~PB} / \mathrm{DMF}$ for $6 \mathrm{~h}$ or $20 \mathrm{mmol} \mathrm{L}^{-1} \mathrm{AP} / \mathrm{DMF}$ for $24 \mathrm{~h}$ to get PB-GP or APGP, respectively, as control samples. Fig. S3a, b show the

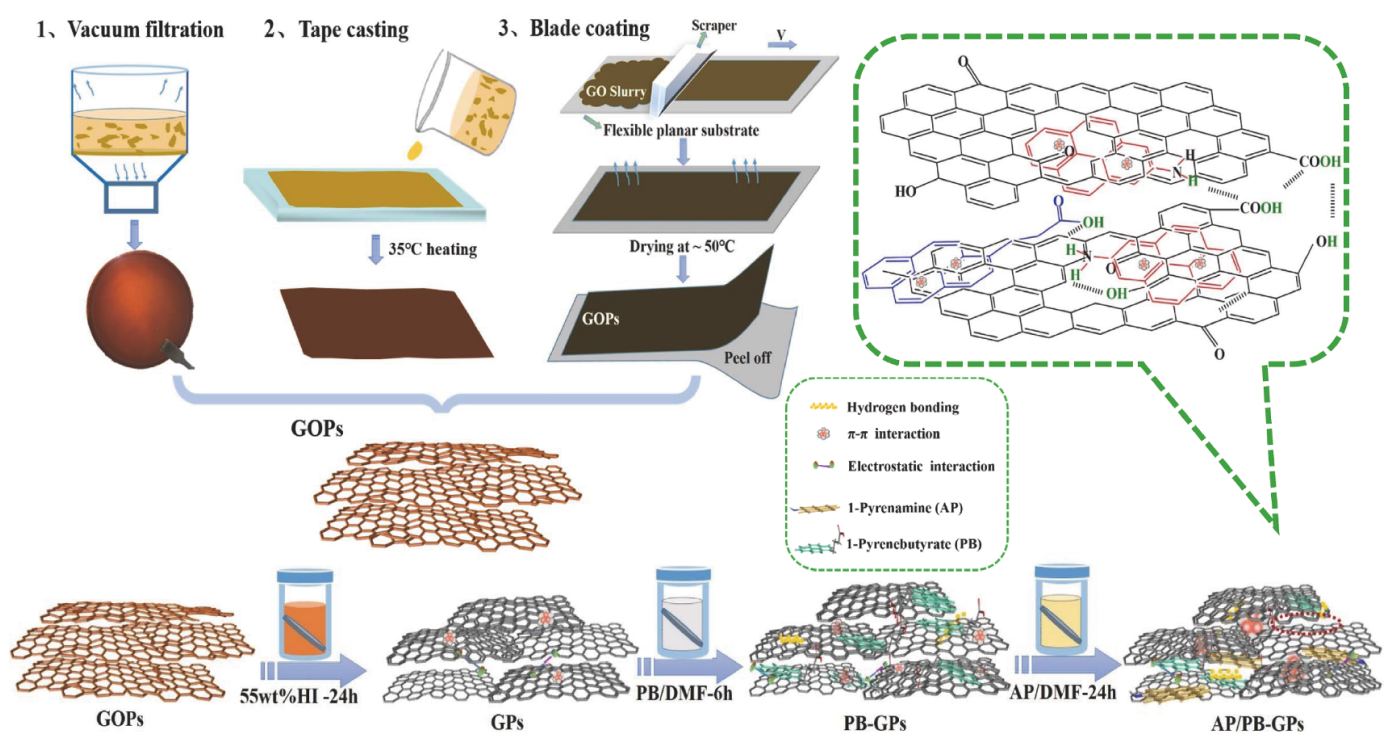

Figure 1 Schematic of fabrication of AP/PB-GPs. 
typical SEM and AFM images of individual GO platelets; the typical thickness of the hydrated GO platelets made by milder oxidation is $\sim 0.83 \mathrm{~nm}$ and the size ranges from several to tens of micrometers (Fig. S3c). After reduction by HI, variety of holes or wavy edges are observed on the platelets (Fig. S3d), which might promote the surface adsorption and anchoring of small molecules. Compared with the RGO platelets, the AP- and AP/PB-decorated platelets have the mean thicknesses of $\sim 1.66$ and $\sim 2.13 \mathrm{~nm}$ (Fig. S3e, f); the higher roughness indicates the spontaneous multilayer adsorption of the conjugated molecules on the graphene platelets $[35,43]$. Thicker AP/ PB-GP was also fabricated by tap-casted GOP and the commercial blade-coated GOP (see more details in the experimental section).

The morphology of AP/PB-GP was characterized based on an optimal AP/PB concentration ratio of 4:1. A highlyordered hierarchical structure with high alignment degree is observed from the cross-sectional SEM image shown in Fig. 2a. The optical image in the inset of Fig. 2a shows a $\sim 4.0-\mathrm{cm}$-diameter, free-standing paper with a shiny metallic luster, which exhibits an impressive flexibility when being folded. The energy dispersive spectroscopy (EDS) mapping taken from the cross-section shows the overall homogeneous distribution of $\mathrm{C}, \mathrm{O}$ and $\mathrm{N}$ in AP/PB-GP, as shown in Fig. 2b. XRD was used to evaluate the as- sembly of the paper and the spectra are shown in Fig. 2c. The characteristic peak at $2 \theta=10.06^{\circ}$ in the spectrum of GOP corresponds to an interplanar spacing of $0.73 \mathrm{~nm}$, smaller than the thickness of hydrated GO platelets measured by AFM $(\sim 0.83 \mathrm{~nm})$. After the reduction by HI, the GP shows a largely reduced interlayer distance of $\sim 0.35 \mathrm{~nm}$, close to that of graphite due to the restored degree of graphitization. When $\mathrm{AP}, \mathrm{PB}$ or $\mathrm{AP} / \mathrm{PB}$ are decorated in graphene platelets, respectively, the hybrid GPs demonstrate interplanar spacings $(\sim 0.37 \mathrm{~nm})$ slightly larger than that of bare GP. But the very little variation of the interlayer spacing suggests that the $\mathrm{AP}$ or $\mathrm{PB}$ molecules are not likely to intercalate into graphene layers, but primarily adsorbed on the edges or defects of graphene platelets [31]. The dramatic difference in layer thickness from XRD or AFM shall be closely related to the different sample preparations for two characterizations. From the Raman spectra (Fig. 2d), D $\left(1345 \mathrm{~cm}^{-1}\right)$ and $G$ $\left(1591 \mathrm{~cm}^{-1}\right)$ bands can be identified from GOP and the downshift of $\mathrm{G}$ band to $1581 \mathrm{~cm}^{-1}$ after $\mathrm{HI}$ reduction shall be caused by the recovery of the hexagonal network of carbon atoms. The increased $I_{\mathrm{D}} / I_{\mathrm{G}}$ ratio is due to the removal of carbon atoms during the reduction process [29]. The AP- or PB-decorated GP exhibits slightly upshifted G-band (to $1587-1591 \mathrm{~cm}^{-1}$ ) compared with that of GP and accompanying the appearance of $\mathrm{D}^{\prime}$ band
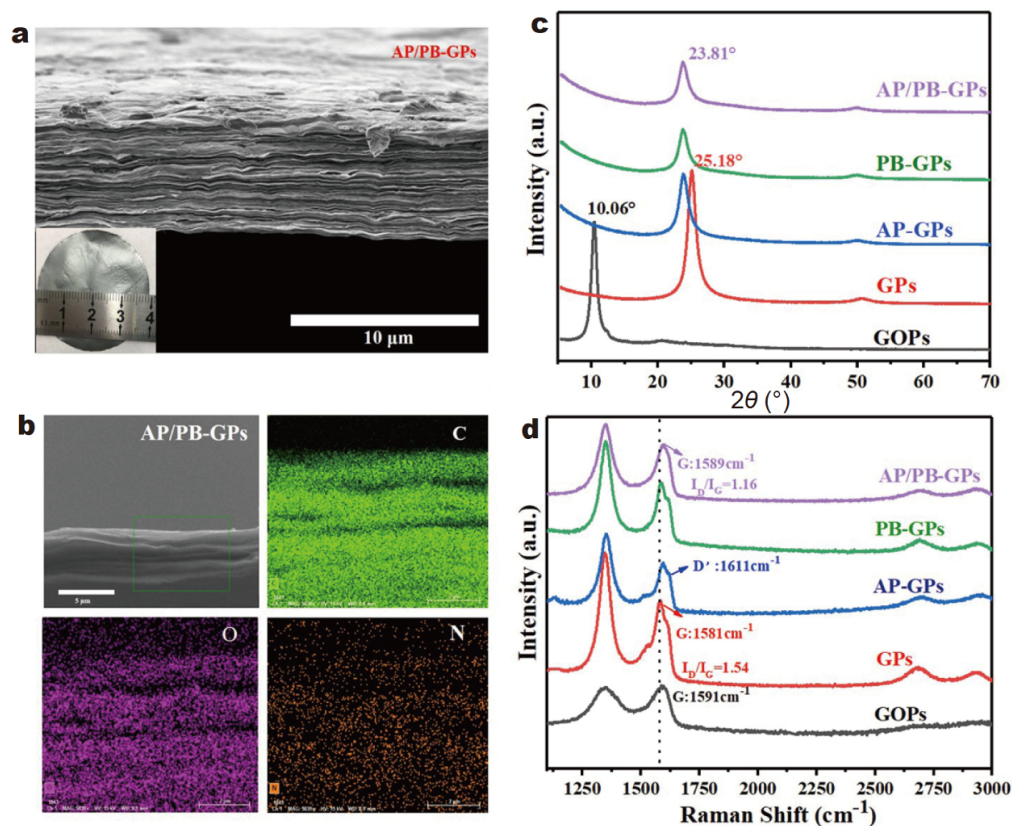
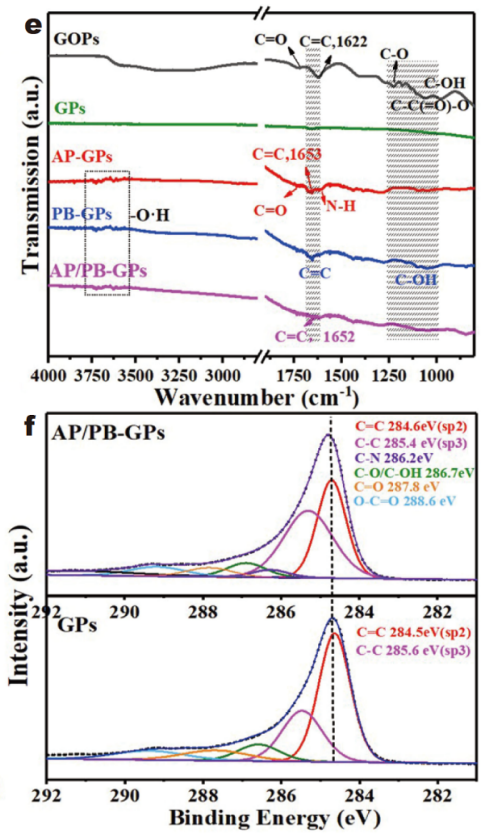

Figure 2 Morphological and structural characterizations of AP/PB-GP. (a) Typical cross-sectional SEM image of AP/PB-GP. Inset shows the optical photograph of a free-standing paper with diameter of $\sim 4.0 \mathrm{~cm}$. (b) SEM image and the corresponding EDS elemental mappings of AP/PB-GP (scale bar: $5 \mu \mathrm{m}$ ). (c) XRD patterns, (d) Raman spectra and (e) FTIR spectra of GOP, GP, AP/GP and AP/PB-GP. (f) XPS C 1s spectra of GP and AP/PB-GP. 
$\left(1611 \mathrm{~cm}^{-1}\right)$, which is likely the consequence of $\pi-\pi$ interactions between aromatic pyrene domain resonance and the disorder arising from the orbital hybridization and charge transfer of the aromatic molecules with graphene [4]. At the same time, the $I_{\mathrm{D}} / I_{\mathrm{G}}$ decreases from 1.54 for GP to 1.16 for AP/PB-GP (more details in Table S2), probably caused by the coverage of pyrene groups onto the defect sites or vacancies of graphene lamellae [44].

From the FTIR spectra shown in Fig. 2e, characteristic stretching vibrations of $\mathrm{O}-\mathrm{H} \quad\left(3500 \mathrm{~cm}^{-1}\right), \quad \mathrm{C}=\mathrm{O}$ $\left(1740 \mathrm{~cm}^{-1}\right)$ and $\mathrm{C}-\mathrm{O}\left(1230 \mathrm{~cm}^{-1}\right)$ groups are observed in GOP. After reduction, the GP exhibits a much weaker infrared absorption resulting from the partial removal of functional groups. The vibration of graphitic domains in GOP shifts from 1622 to $1652 \mathrm{~cm}^{-1}$ in neat or crosslinked GP caused by the improved $\pi-\pi$ interactions [26]. Note that the $-\mathrm{NH}_{2}$ directly linked to pyrene on AP usually does not react with $-\mathrm{COOH}$ on $\mathrm{PB}$ at ambient condition due to its low reactivity [45]; the H-bonding interaction mainly exists between AP or $\mathrm{PB}$ and graphene platelets. For AP- or AP/PB-decorated GPs, the vibration around $1640 \mathrm{~cm}^{-1}$ corresponds to the stretching of $\mathrm{N}-\mathrm{H}$ amide groups; the broad C-OH stretching at $3549,3619,3620$ or $3695 \mathrm{~cm}^{-1}$ indicates the H-bonding interactions exist between the conjugated molecules and graphitic lamellae contributed from groups of $-\mathrm{COOH}, \mathrm{O}-\mathrm{C}=\mathrm{O}$ or $-\mathrm{OH}$ (on the basal plane or at the edge) [31]. XPS C 1s spectra in Fig. $2 \mathrm{f}$ show that the reduction has largely restored the graphitic structure, indicated by the peak at around $\sim 284.6 \mathrm{eV}$ in GP when being compared with those of GOP (Fig. S4). Meanwhile, the signals arising from the epoxide and hydroxyl moieties at $286.7 \mathrm{eV}$ are greatly reduced in GP, coinciding with the decrease in the O 1s/C 1s atomic ratio from 0.32 to 0.12 (Table S2), whereas a small fraction of carboxyl and carbonyl groups remain in GP. The residual carboxyl functional groups located in the edges or defects were difficult to be removed by HI [42], which provides active sites for hydrogen bonding interaction. For the AP-, PB- or AP/PB-crosslinked GPs, the $\mathrm{C}=\mathrm{C} / \mathrm{C}-\mathrm{C}$ peak slightly upshifts from 284.5 to $284.8 \mathrm{eV}$, which is likely due to the $\pi-\pi$ interaction between the conjugated pyrene and graphene platelets [46].

The mechanical merits of the as-prepared paper were evaluated by uniaxial tensile tests performed in the atmosphere and the typical stress-strain curves plotted in Fig. 3a are selected from those in Fig. S5. The averaged breaking strength of GP (311 $\pm 42 \mathrm{MPa})$ is much higher than that of GOP $(138 \pm 16 \mathrm{MPa})$ due to $\pi-\pi$ interactions in the stacking between adjacent graphene lamellae after most oxygen-containing groups are removed along with a decreased interlayer distance. The effective interlayer load transfer at high strains $(\sim 10 \%$ in partial tensile data (Fig. S5)) needs strong interlayer interaction, which however is not found in GOP due to the large interlayer
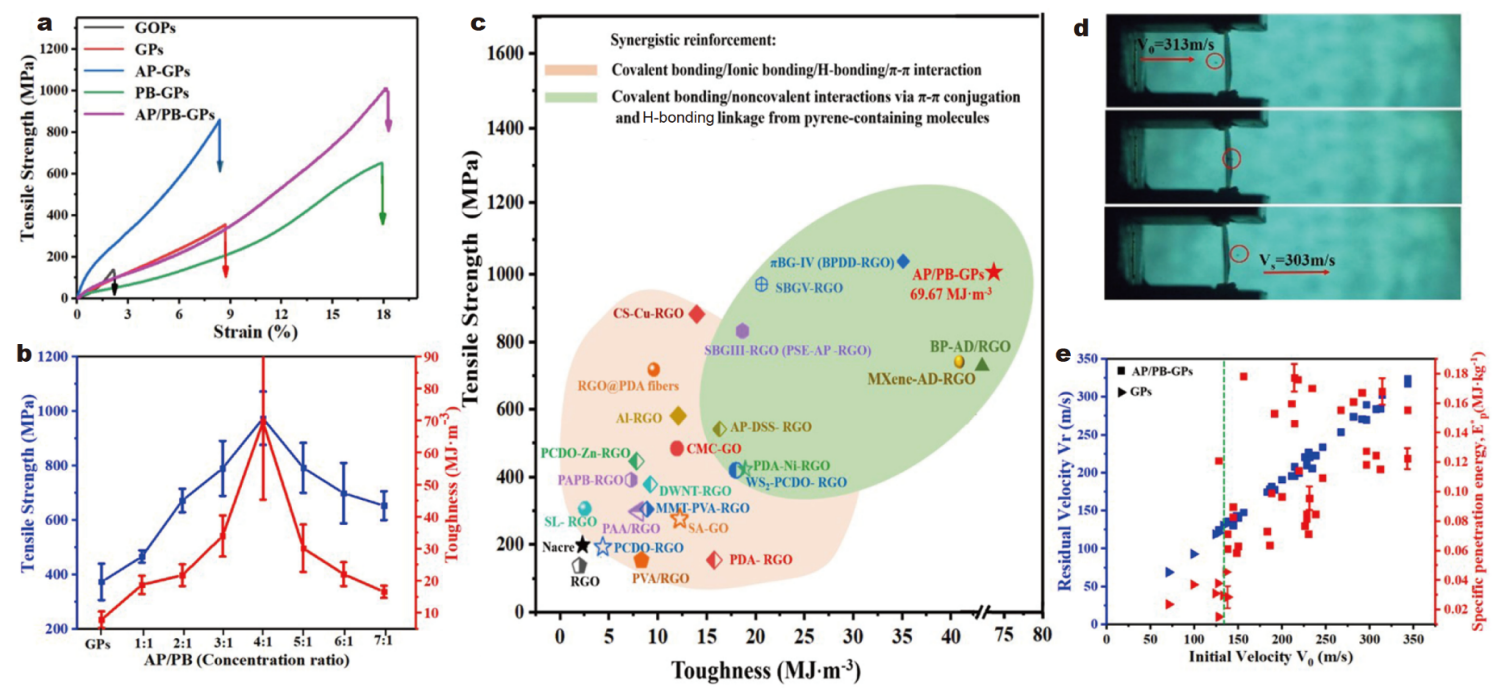

Figure 3 Mechanical and impact performances of AP/PB-GP. (a) Typical stress-strain curves for GOP, GP, and AP or/and PB crosslinked GP. (b) Strength and toughness for AP/PB-GP depending on the AP/PB concentration ratio; the averaged strength and toughness values were obtained by several tensile curves from the same crosslinked graphene papers. (c) Comparison of ultimate strength and failure strain of AP/PB-GP with reported RGO-based hybrid papers via various cross-linking strategies. The values are listed in Table S4 with references. (d) Optical images of 25- $\mu$ m-thick AP/ PB-GP in gas gun ballistic impact test, snapshotted by the high-speed camera. (e) Impact residual velocity $\left(V_{\mathrm{r}}\right)$ and specific penetration energy $\left(E_{\mathrm{p}}^{*}\right)$ as a function of initial velocity $\left(V_{0}\right)$ obtained from impact tests. 
distance $[37,47]$. As listed in Table S3, AP-GP provides an impressive averaged tensile strength $(836 \pm 24 \mathrm{MPa})$ and Young's modulus $(14.1 \pm 0.58 \mathrm{GPa})$, much higher than bare GP or PB-GP. It is worth noting that the PB-GP shows substantially increased toughness (30.08 \pm $1.4 \mathrm{MJ} \mathrm{m}^{-3}$ ) with a prolonged failure strain of $\sim 18.1 \%$ (Fig. S6a). As a result of binary crosslinking, AP/PB-GP with the optimum $\mathrm{AP} / \mathrm{PB}$ concentration ratio of 4:1 demonstrates an unprecedentedly combination of averaged high tensile strength $(974 \pm 98 \mathrm{MPa})$ and record-high toughness $\left(69.67 \pm 15.3 \mathrm{MJ} \mathrm{m}^{-3}\right)$, nearly 2.4 and 7.3 fold higher than bare GP. The strength and toughness monotonically increase with the AP/PB concentrationratio of 4:1 while keeping the immersion durations unchanged, but further increase in the AP concentration leads to decrease in the performances (Fig. 3b, Fig.S6b, c). The excess amount of AP molecules would cause a spontaneous agglomeration that hardly forms adequate bridging interface (Fig. S6b-e), leading to a stress concentration and failure upon loading [48]. The comparison between the ultimate tensile strength and strain of AP/ PB-GP with those of natural nacre, RGO and other crosslinked RGO paper is summarized in Fig. $3 c$, and the mechanical values are listed in Table S3. As we can see, compared with nacre-mimetic binary/ternary crosslinked types based on the hydrogen, ionic, and $\pi-\pi$ interactions in orange area (Fig. 3c), the AP/PB-GP with the optimum $\mathrm{AP} / \mathrm{PB}$ concentration ratio has achieved a superior integration of tensile strength and toughness, noticeably surpassing those reported pyrene-modified graphene papers including PSE-AP-RGO [29], MrGO-AD [30], and rGO/BP-AD [33]. As shown in Fig. S7, the thicker AP/ PB-GP $(\sim 18 \mu \mathrm{m})$ made by tap casting (named as AP/PBTGP) demonstrates an averaged tensile strength of $411 \pm 46 \mathrm{MPa}$ together with a toughness of $15.19 \pm$ $1.3 \mathrm{MJ} \mathrm{m}^{-3}$, which is inferior to those of AP/PB-GP, indicating the importance of assembly. The $60-\mu \mathrm{m}$-thick AP/PB-GP made by commercial blade coating (AP/PBCGP) maintains a fairly high averaged strength of $451 \pm$ $12 \mathrm{MPa}$ and an impressive toughness of $21.1 \pm$ $2.3 \mathrm{MJ} \mathrm{m}^{-3}$. Further extension of this cross-linking strategy may allow a variety of high-quality GP with tunable mechanical properties.

The dynamic impact resistance of AP/PB-GP with different thicknesses $(25-80 \mu \mathrm{m})$ were evaluated with microscale ballistic impact testing using a steel ball (200 $\mu \mathrm{m}$ in diameter) moving at a speed of approximately $350 \mathrm{~m} \mathrm{~s}^{-1}$ (Fig. 3d). The residual velocity $\left(V_{\mathrm{r}}\right)$ and specific penetration energy $\left(E_{\mathrm{p}}^{*}\right)$ are plotted in Fig. $3 e$ as a function of initial velocity $\left(V_{0}\right)$. The characteristic antiballistic performance of materials can be defined as the specific energy absorption: $E_{\mathrm{p}}^{*}=1 / 2 m\left(V_{0}^{2}-V_{\mathrm{r}}^{2}\right) \times\left(\rho A_{\mathrm{s}} h\right)^{-1}$, where $m$ is the mass of the projectile, $\rho$ is the density of the specimen, $h$ is the specimen thickness and $A_{\mathrm{s}}$ is the area of a strike face $\left(A_{\mathrm{s}}=\pi D^{2} / 4\right.$, strike face diameter $\left.D>h\right)$ [34]. As we can see, the bare $25-\mu \mathrm{m}$-thick GP could not withstand high-speed impact $\left(V_{0} \sim 150 \mathrm{~m} \mathrm{~s}^{-1}\right)$, evidenced by the penetration energy $\left(E_{\mathrm{p}}\right)$ close to zero $\left(V_{0} \approx V_{\mathrm{r}}\right.$, $\left.E_{\mathrm{p}}=1 / 2 m\left(V_{0}^{2}-V_{\mathrm{r}}^{2}\right)\right)$. In contrast, the $25-\mu \mathrm{m}$-thick binary crosslinked GP shows noticeably higher $E_{\mathrm{p}}^{*}$ $\left(\sim 0.17 \mathrm{MJ} \mathrm{kg}^{-1}\right)$ especially for high impact velocities $\left(V_{0} \approx\right.$ $313 \mathrm{~m} \mathrm{~s}^{-1}$ ), nearly 5.6 fold higher than those of bare GPs $\left(\sim 0.03 \mathrm{MJ} \mathrm{kg}^{-1}\right)$, outperforming steel, ductile alumina and amorphous glassy polymer $[34,36,49]$. Specifically, as the thickness was scaled up to $\sim 80 \mu \mathrm{m}$ (Fig. S8), the binary crosslinked papers still can bear impact efficiently with relatively stable $E_{\mathrm{p}}^{*}\left(\sim 0.16 \mathrm{MJ} \mathrm{kg}^{-1}, V_{0} \approx 350 \mathrm{~m} \mathrm{~s}^{-1}\right.$, Fig. S8a), showing the potential scalability. After penetration, the fracture of GP shows curled edge perforation with small fragmentation, suggesting that the lamellae in GP have experienced immediate shear penetration and breakage of sheets at the impact site and shown reluctance to energy absorption, which lead to the delamination and more destructive cracks (Fig. $S 9 a_{1}-d_{1}$ ). While in the AP/PB-GP, on the other hand, the lamellae display tortuous feature of breakage with smaller cracks than that in the bare GP for the same initial velocity, initiating more plastic void growth and crack deflections under higher impact velocity (Fig. S9b-d).

The electrical conductivities of graphene papers were measured with a standard four-probe method and summarized in Tables S4, S5. Due to the restoration of $\pi-\pi$ conjugation and closer stacking between layers, the electrical conductivity of GP has been greatly improved to $492 \pm 39 \mathrm{~S} \mathrm{~cm}^{-1}$, in accordance with previously reported results $[31,35]$. Notably, the conductivity of PB-GP further increases to $673 \pm 17.3 \mathrm{~S} \mathrm{~cm}^{-1}$, which well rationalizes the understanding that the pyrene moiety facilitates the charge transfer by strong adhesion with the graphitic plane via $\pi-\pi$ interaction $[35,50]$. It is worth noting that the conductivities of fractured papers are typically reduced after uniaxial tensile tests, which may be explained by the fact that graphene lamellar sliding, deformation, or even partial fracture, generate unfavorable effects on the electrical conductance.

Strain-dependent Raman provides the difference in stress transfer for bare GP and AP/PB-GP in the intermediate plastic deformation stage (with strain of $2 \%-7 \%$ ). As shown in Fig. 4a and Fig. S10, the domain mapping of bare GP taken from six spots shows a stable G-band 
frequency at a strain of up to $\sim 7 \%$. In contrast, the AP/ PB-GP shows more obvious dependence of Raman shift on the strain, evidenced by a downshift of $G$ peak from
$1591 \mathrm{~cm}^{-1}$ (for $\sim 2 \%$ strain) to $1583 \mathrm{~cm}^{-1}$ (for $\sim 7 \%$ strain). The strain-sensitive Raman indicates the more efficient stress transfer to graphene [51]. XRD was employed on
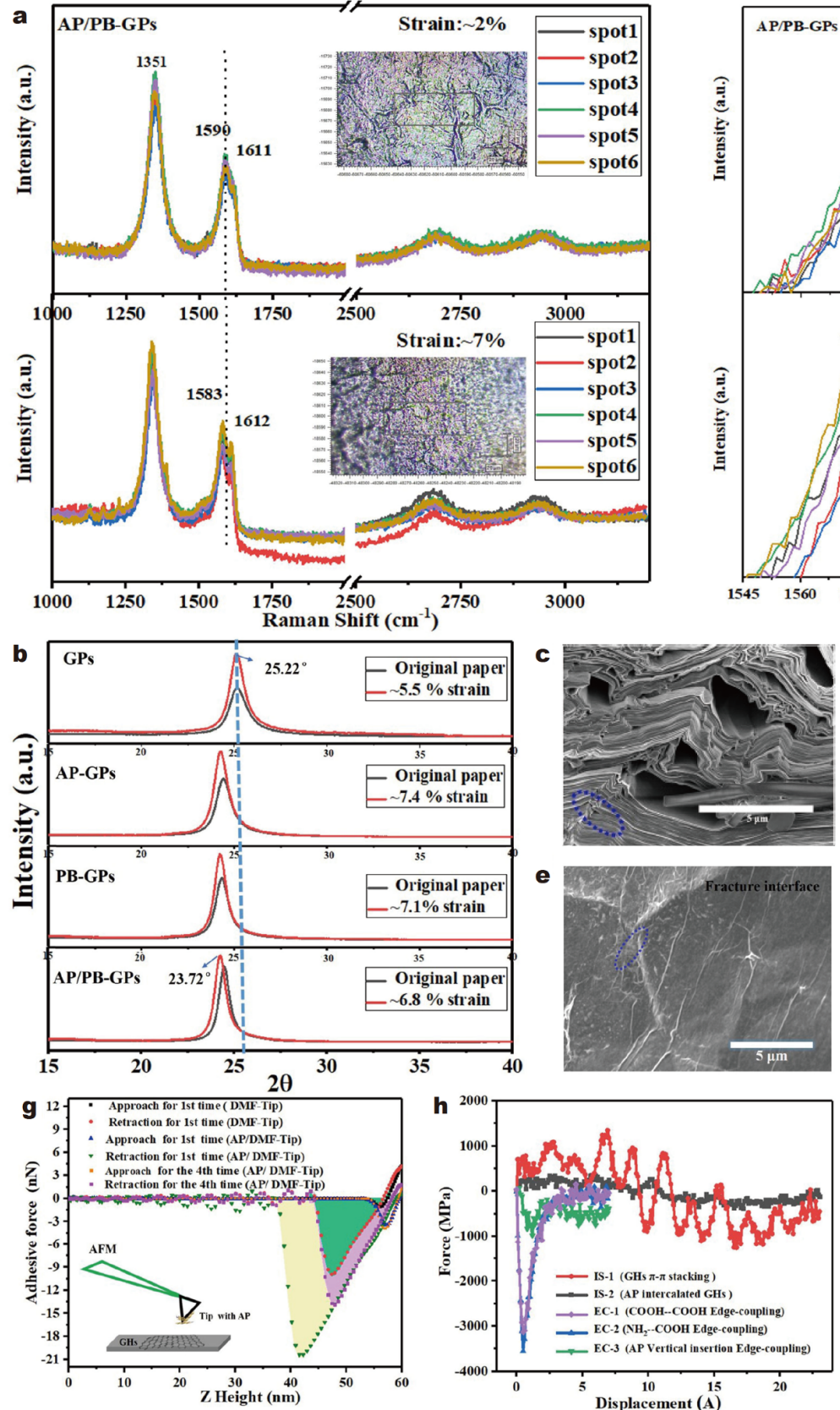
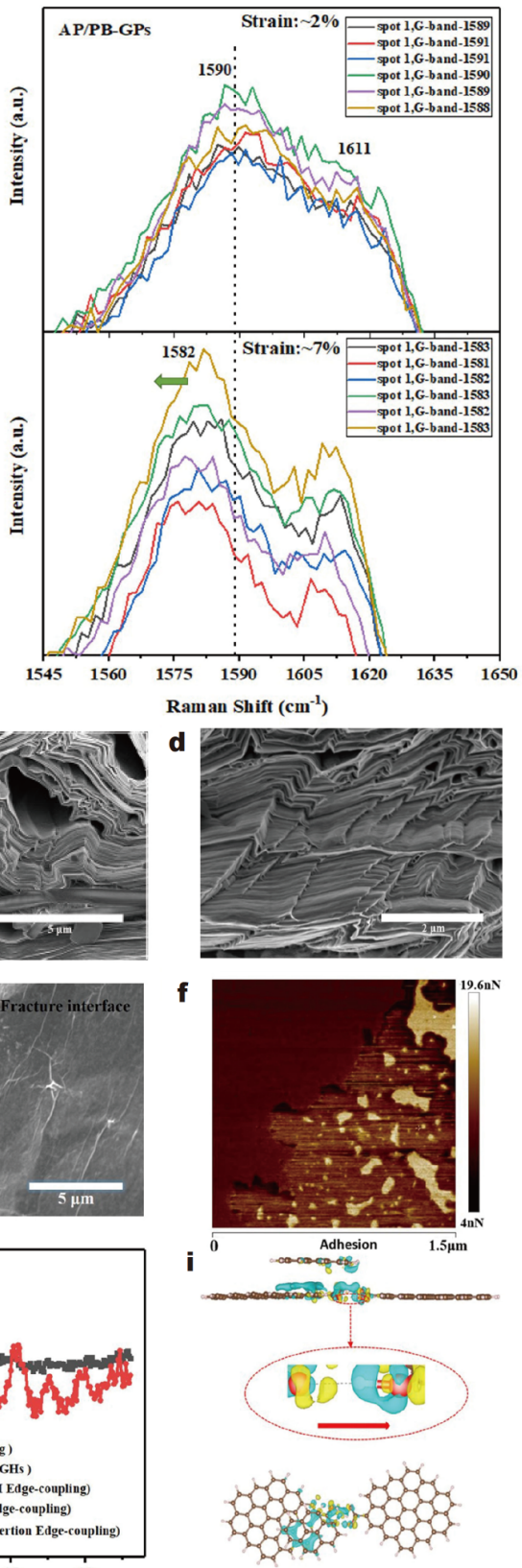

Figure 4 Fracture mechanism of AP/PB-GP. (a) Strain-dependent Raman spectra taken from six spots in domain mapping area: inset optical images show the spatial mapping $\left(\sim 200 \mu \mathrm{m}^{2}\right.$ square area) and the figure on the right represents the local enlargement of G peak. (b) XRD patterns of the GP strips before or during a stretching applied (without breaking). (c) Typical cross-sectional SEM image of fractured AP/PB-GP and (d) zoomed in the areas labeled in (c). (e) SEM image of wrinkled internal surface morphology. (f) Adhesion force mapping of the tip with AP modification approaching the graphene sheets for the first time. (g) Adhesion force measurement between AP-modified tips and individual graphene platelets; inset shows the schematic of setup. (h) DFT simulated curves of force versus displacement for various interlayer coupling mechanisms. (i) Optimized atomic structure of adsorption of an AP molecule near- $\mathrm{COOH}$ groups and its iso surface of difference charge density $\Delta \rho$. 
monitoring the structure change in-situ in the intermediate plastic deformation stage (strain of 5\%-7\%). As we can see from Fig. $4 \mathrm{~b}$ and Table S6, upon a strain applied, the (002) diffraction peak of the single or binary hybrid papers slightly moves to small angles; the full width at half maximum (FWHM) becomes larger, in contrast to bare GP. The broader distribution of the distance can be explained by the sequential breakage of sacrificing hydrogen bonds under strains; the graphene nanosheets begin to slide over each other, initiating the crack to detach the lamellae orientation. After breakage, the microscopic feature was checked with SEM and the typical images are shown in Fig. $4 \mathrm{c}-\mathrm{e}$. The fracture crosssection studies show that the bare GP presents a loose pull-out fracture feature with curled edges (Fig. S11 $1 \mathrm{a}_{1}$ ). For the PB-GP, in part, PB molecule would bridge with graphene via the hydrogen bonding between the negatively charged carboxylate on the side alkyl chain and residual groups in graphene sheets, providing high resistance toward crack propagation, and thus the graphene sheets tend to rebound at the breaking, resulting in highly resilient fracture without those curled edges (Fig. $S 11 c_{1}$ ). In addition, the partial misalignment of PB (Fig. S11 $c_{3}$, marked by blue ovals) adhered to the graphene inner surface may deflect the advancing crack by sliding under high stress, thereby increasing crack area to prevent premature failure [52]. From the Fig. $S 9 a_{2}-d_{2}$, the platelets packed more uniformly and tighter in the fracture of AP-GP, AP/PB-GP, and the voids also further reduced in lamellar. Furthermore, the fractured rectangular tensile strip of the binary hybrid papers (Fig. S12) exhibits curly elongated shape and more tortuous resilient breakage lines are accompanied by local tiny delaminated cavities (Fig. 4d), indicating that the local stress/strain incompatibility prefers to form stress concentrations at the interface under destructive stress. In addition, from Fig. 4 e and Fig. $\mathrm{S}_{11} \mathrm{a}_{3}-\mathrm{d}_{3}$, we can see that single or binary decorated papers demonstrate smooth and adhesive wrinkled interface topologies, which distinguishes them from bare GP.

The macroscopic mechanical property shall reflect the collective behavior of factors like intrinsic hierarchical structures, interface adhesion and synergy of multiple toughening effects $[53,54]$. Since the AP-GP displayed outstanding averaged tensile strength $(836 \pm 24 \mathrm{MPa})$ close to that of AP/PB-GP, AFM tips have been modified with AP molecules to reveal the role of interface on the load transfer and interfacial slippage. The surface force mapping shown in Fig. $4 \mathrm{f}$ reveals that the adhesion forces near the defective cavities or wavy edges (observed in
HRTEM in Fig. S13) are obviously higher than those in aromatic domains. Fig. $4 \mathrm{~g}$ shows the curves of adhesion force versus height, in which the area marked by color represents the energy required to pull the tip apart the contact. As can be seen from the curves, AP-modified tips exhibit a dramatic enhancement in adhesion energy marked by yellow when being compared with the bare AFM tip marked by green in the same DMF solvent, indicating the strong interactions mainly dominated by $\pi$ $\pi$ stacking and H-bonding between graphene platelets and AP molecules. In the fourth testing cycle, the adhesion energy shown in the purple area gradually decreases to a degree close to that for the bare AFM tip, indicating that most AP molecules have been separated from the tip due to strong adhesion with graphene.

To search the favorable adsorption sites on graphene, the $\mathrm{H}$-bonding interaction between $\mathrm{AP}$ or $\mathrm{PB}$ and graphene platelets has been investigated via DFT calculations. The energy optimization (Table S1) suggests that weaker $\mathrm{H}$-bonding exists between $\mathrm{AP}$ and $\mathrm{PB}$ molecules due to the existence of electron donor group at the edge of PB molecules. However, both of them have strong coupling effects on the edge carboxyl groups of graphene; the H-bonding mainly distributes among amine-terminated AP, carboxyl-terminated $\mathrm{PB}$ and polar functional groups $(-\mathrm{COOH},-\mathrm{OH})$ at the edges of graphene platelets. Herein, two main stretching mechanisms, IS and EC, have been considered between AP molecules and graphene sheets; the detailed methods and modelling are described in the simulation methods. The simulated stretching force versus distance curves for different IS or EC mechanisms are shown in Fig. 4h. For the inter-layer slipping mechanism, the optimized models include two graphene platelets directly coupled with $\pi-\pi$ conjunction (denoted as IS-1, Fig. S1a) and interlayer spacing laterally with inserted AP (IS-2, Fig. S1b). The simulation shows that the inter-layer slipping strength of two graphene platelets is slightly weakened through the insertion of AP molecule, suggesting that the interlayer slipping mechanism is not the dominant factor in strength enhancement. The fluctuation of the IS-1 curve in Fig. $4 \mathrm{~h}$ originates from the repeated coupling and decoupling of $\pi-\pi$ conjunction in two adjacent graphene platelets. For the edge-coupling mechanism, a comprehensive calculated analysis in Fig. S2 demonstrates that two $-\mathrm{COOH}$ groups coupled from edges correspond to the highest stretching strength; the AP position, i.e., at the edge or in the interior of aromatic domains, has a strong influence on the stretching strength.

Three possible scenarios have been further considered: 
edge coupling by - $\mathrm{COOH}$ of two adjacent platelets (EC-1, Fig. S1c); an AP molecule adsorbed by $-\mathrm{COOH}$ groups nearby (EC-2, Fig. S1d); an AP molecule vertically inserted between two adjacent - $\mathrm{COOH}$ groups (EC-3, Fig. S1e). As shown in Fig. 4h, the adsorption of AP parallel to the adjacent platelets (EC-2) increases the tensile strength, while the vertical insertion (EC-3) deteriorates the tensile strength. In addition, the edge-coupling mechanism yields a much bigger stretching strength than the inter-layer slipping mechanism, indicating that the edge-coupling is probably the dominant mechanism when the AP-GP is stretched. To further investigate the origin for the edge-coupling in the enhancement of stretching strength, the charge differential density $(\Delta \rho)$ for the AP adsorption was calculated. As shown in Fig. $4 \mathrm{i}$, the oxygen atom in the $-\mathrm{COOH}$ group gains charge while the connecting hydrogen atom in the other $-\mathrm{COOH}$ group losts charge upon the adsorption of AP, leading to an amplified electric dipole moment and contributing to the enhancement by the electric dipole interaction. Through such interactions, the individual graphene platelets are interlinked via hydrogen bonds mediated by edge $-\mathrm{COOH}$ groups and cross-linked molecules.

\section{CONCLUSIONS}

In summary, we fabricated $\mathrm{AP} / \mathrm{PB}$ binary cross-linked graphene papers via a straightforward immersion strategy. The two types of pyrene-terminal small molecules with rigidity (AP) and flexibility (PB) interpenetrated into graphene layers or densely packed galleries to form robust interface. Consequently, the AP/PB-GP exhibited a combination of simultaneous enhancement in strength, toughness and electrical conductivity, allowing its application in impact resistance. Adhesion, structural and fracture characteristic analyses as well as DFT simulations further revealed that the interfacial sacrificial bonds including $\pi-\pi$ stacking interaction between aromatic elements and $\mathrm{H}$-bonding between polar functional groups are crucial for the strong interface adhesion. Meanwhile, the wrinkled and defective pores within graphene platelets ensure the favorable adhesion of the pyrene molecules and imperatively maximize the interfacial binding between lamellae, facilitating deflecting crack and plastic deformation under loading. Our research provides a feasible strategy and fundamental insight for the strong yet ductile graphene papers and related mechanisms, which may shed light on the design of high impact-tolerance papers for emerging applications, such as lightweight protective shields, flexible energy storage devices, intelligent generators and other electronic devices.

Received 6 July 2020; accepted 18 September 2020;

published online 16 December 2020

1 Lee C, Wei X, Kysar JW, et al. Measurement of the elastic properties and intrinsic strength of monolayer graphene. Science, 2008, 321: $385-388$

2 Zhao H, Min K, Aluru NR. Size and chirality dependent elastic properties of graphene nanoribbons under uniaxial tension. Nano Lett, 2009, 9: 3012-3015

3 Li YQ, Yu T, Yang TY, et al. Bio-inspired nacre-like composite films based on graphene with superior mechanical, electrical, and biocompatible properties. Adv Mater, 2012, 24: 3426-3431

4 Moon IK, Lee J, Ruoff RS, et al. Reduced graphene oxide by chemical graphitization. Nat Commun, 2010, 1: 73

5 Chen $\mathrm{H}$, Müller MB, Gilmore KJ, et al. Mechanically strong, electrically conductive, and biocompatible graphene paper. Adv Mater, 2008, 20: 3557-3561

6 Zhu Y, Ji H, Cheng HM, et al. Mass production and industrial applications of graphene materials. Natl Sci Rev, 2017, 5: 90-101

7 Wang B, Cunning BV, Park SY, et al. Graphene coatings as barrier layers to prevent the water-induced corrosion of silicate glass. ACS Nano, 2016, 10: 9794-9800

8 Ci S, Cai P, Wen Z, et al. Graphene-based electrode materials for microbial fuel cells. Sci China Mater, 2015, 58: 496-509

9 Kong W, Kum H, Bae SH, et al. Path towards graphene commercialization from lab to market. Nat Nanotechnol, 2019, 14: 927-938

10 Yao HB, Fang HY, Tan ZH, et al. Biologically inspired, strong, transparent, and functional layered organic-inorganic hybrid films. Angew Chem Int Ed, 2010, 49: 2140-2145

11 Lin X, Shen X, Zheng Q, et al. Fabrication of highly-aligned, conductive, and strong graphene papers using ultralarge graphene oxide sheets. ACS Nano, 2012, 6: 10708-10719

12 Shin MK, Lee B, Kim SH, et al. Synergistic toughening of composite fibres by self-alignment of reduced graphene oxide and carbon nanotubes. Nat Commun, 2012, 3: 650

13 Zhang Y, Gong S, Zhang Q, et al. Graphene-based artificial nacre nanocomposites. Chem Soc Rev, 2016, 45: 2378-2395

14 Li P, Yang M, Liu Y, et al. Continuous crystalline graphene papers with Gigapascal strength by intercalation modulated plasticization. Nat Commun, 2020, 11: 2645

15 Wang J, Cheng Q, Tang Z. Layered nanocomposites inspired by the structure and mechanical properties of nacre. Chem Soc Rev, 2012, 41: 1111-1129

16 Gong $\mathrm{S}, \mathrm{Ni} \mathrm{H}$, Jiang $\mathrm{L}$, et al. Learning from nature: constructing high performance graphene-based nanocomposites. Mater Today, 2017, 20: 210-219

17 Song $\mathrm{P}, \mathrm{Xu} \mathrm{Z}, \mathrm{Wu} \mathrm{Y}$, et al. Super-tough artificial nacre based on graphene oxide via synergistic interface interactions of $\pi-\pi$ stacking and hydrogen bonding. Carbon, 2017, 111: 807-812

18 Xia S, Wang Z, Chen H, et al. Nanoasperity: structure origin of nacre-inspired nanocomposites. ACS Nano, 2015, 9: 2167-2172

19 Ming P, Song Z, Gong S, et al. Nacre-inspired integrated nanocomposites with fire retardant properties by graphene oxide and montmorillonite. J Mater Chem A, 2015, 3: 21194-21200

20 Ma T, Gao HL, Cong HP, et al. A bioinspired interface design for improving the strength and electrical conductivity of graphenebased fibers. Adv Mater, 2018, 30: 1706435 
21 Chen K, Shi B, Yue Y, et al. Binary synergy strengthening and toughening of bio-inspired nacre-like graphene oxide/sodium alginate composite paper. ACS Nano, 2015, 9: 8165-8175

22 Shahzadi K, Zhang X, Mohsin I, et al. Reduced graphene oxide/ alumina, a good accelerant for cellulose-based artificial nacre with excellent mechanical, barrier, and conductive properties. ACS Nano, 2017, 11: 5717-5725

23 Cheng Y, Peng J, Xu H, et al. Glycera-inspired synergistic interfacial interactions for constructing ultrastrong graphene-based nanocomposites. Adv Funct Mater, 2018, 28: 1800924

24 Yang $\mathrm{M}, \mathrm{Xu} \mathrm{Z}$, Li P, et al. Interlayer crosslinking to conquer the stress relaxation of graphene laminated materials. Mater Horiz, 2018, 5: 1112-1119

25 Dhar P, Phiri J, Szilvay GR, et al. Genetically engineered protein based nacre-like nanocomposites with superior mechanical and electrochemical performance. J Mater Chem A, 2020, 8: 656-669

26 Chen $\mathrm{Z}, \mathrm{Lu} \mathrm{H}$. Constructing sacrificial bonds and hidden lengths for ductile graphene/polyurethane elastomers with improved strength and toughness. J Mater Chem, 2012, 22: 12479-12490

27 Liu S, Liu J, Xu Z, et al. Artificial bicontinuous laminate synergistically reinforces and toughens dilute graphene composites. ACS Nano, 2018, 12: 11236-11243

28 Cui W, Li M, Liu J, et al. A strong integrated strength and toughness artificial nacre based on dopamine cross-linked graphene oxide. ACS Nano, 2014, 8: 9511-9517

29 Wan S, Li Y, Mu J, et al. Sequentially bridged graphene sheets with high strength, toughness, and electrical conductivity. Proc Natl Acad Sci USA, 2018, 115: 5359-5364

30 Zhou T, Wu C, Wang Y, et al. Super-tough MXene-functionalized graphene sheets. Nat Commun, 2020, 11: 2077

31 Wan S, Chen Y, Wang Y, et al. Ultrastrong graphene films via long-chain $\pi$-bridging. Matter, 2019, 1: 389-401

32 Zhang Y, Peng J, Li M, et al. Bioinspired supertough graphene fiber through sequential interfacial interactions. ACS Nano, 2018, 12: 8901-8908

33 Zhou T, Ni H, Wang Y, et al. Ultratough graphene-black phosphorus films. Proc Natl Acad Sci USA, 2020, 117: 8727-8735

34 Lee JH, Loya PE, Lou J, et al. Dynamic mechanical behavior of multilayer graphene via supersonic projectile penetration. Science, 2014, 346: 1092-1096

$35 \mathrm{Xu} \mathrm{Y,} \mathrm{Bai} \mathrm{H,} \mathrm{Lu} \mathrm{G,} \mathrm{et} \mathrm{al.} \mathrm{Flexible} \mathrm{graphene} \mathrm{films} \mathrm{via} \mathrm{the} \mathrm{filtration} \mathrm{of}$ water-soluble noncovalent functionalized graphene sheets. J Am Chem Soc, 2008, 130: 5856-5857

36 Xie W, Tadepalli S, Park SH, et al. Extreme mechanical behavior of nacre-mimetic graphene-oxide and silk nanocomposites. Nano Lett, 2018, 18: 987-993

37 Wen $\mathrm{Y}, \mathrm{Wu} \mathrm{M}$, Zhang $\mathrm{M}$, et al. Topological design of ultrastrong and highly conductive graphene films. Adv Mater, 2017, 29: 1702831

38 Perdew JP. Density-functional approximation for the correlation energy of the inhomogeneous electron gas. Phys Rev B, 1986, 33: 8822-8824

39 Perdew JP, Burke K, Ernzerhof M. Generalized gradient approximation made simple. Phys Rev Lett, 1996, 77: 3865-3868

40 Grimme S. Semiempirical GGA-type density functional constructed with a long-range dispersion correction. J Comput Chem, 2006, 27: 1787-1799

41 Monkhorst HJ, Pack JD. Special points for Brillouin-zone integrations. Phys Rev B, 1976, 13: 5188-5192

42 Pei S, Zhao J, Du J, et al. Direct reduction of graphene oxide films into highly conductive and flexible graphene films by hydrohalic acids. Carbon, 2010, 48: 4466-4474

43 Kovtyukhova NI, Ollivier PJ, Martin BR, et al. Layer-by-layer assembly of ultrathin composite films from micron-sized graphite oxide sheets and polycations. Chem Mater, 1999, 11: 771-778

$44 \mathrm{Ni} \mathrm{H}, \mathrm{Xu}$ F, Tomsia AP, et al. Robust bioinspired graphene film via $\pi-\pi$ cross-linking. ACS Appl Mater Interfaces, 2017, 9: 2498724992

45 Donckt EV, Dramaix R, Nasielski J, et al. Photochemistry of aromatic compounds. Part 1.-Acid-base properties of singlet and triplet excited states of pyrene derivatives and aza-aromatic compounds. Trans Faraday Soc, 1969, 65: 3258-3262

46 Su Q, Pang S, Alijani V, et al. Composites of graphene with large aromatic molecules. Adv Mater, 2009, 21: 3191-3195

47 Mao L, Park H, Soler-Crespo RA, et al. Stiffening of graphene oxide films by soft porous sheets. Nat Commun, 2019, 10: 3677

48 Suresh S. Graded materials for resistance to contact deformation and damage. Science, 2001, 292: 2447-2451

49 Saghafi H, Fotouhi M, Minak G. Improvement of the impact properties of composite laminates by means of nano-modification of the matrix-a review. Appl Sci, 2018, 8: 2406

50 Dong X, Fu D, Fang W, et al. Doping single-layer graphene with aromatic molecules. Small, 2009, 5: 1422-1426

51 Mohiuddin TMG, Lombardo A, Nair RR, et al. Uniaxial strain in graphene by Raman spectroscopy: $G$ peak splitting, Grüneisen parameters, and sample orientation. Phys Rev B, 2009, 79: 205433

52 Faber KT, Evans AG. Crack deflection processes-I. Theory. Acta Metall, 1983, 31: 565-576

$53 \mathrm{Lu} \mathrm{H}$, Chen Z, Ma C. Bioinspired approaches for optimizing the strength and toughness of graphene-based polymer nanocomposites. J Mater Chem, 2012, 22: 16182-16190

54 Ji D, Kim J. Bioinspired design and fabrication of polymer composite films consisting of a strong and stiff organic matrix and microsized inorganic platelets. ACS Nano, 2019, 13: 2773-2785

Acknowledgements This work was supported by the National Natural Science Foundation of China (51772282, 51972299), and funding from Hefei Center for Physical Science and Technology.

Author contributions Yuan $\mathrm{H}$ conceived the idea, performed the material preparation and wrote the manuscript. Ge L and Ni K performed theoretical calculations. Kan X, Chen SM, Gao M and Pan F helped to carried out the mechanical tensile and impact experiments. Ye J, Xu F, Shu N and Li J contributed to the characterization data analyses. Suo $\mathrm{T}$ and $\mathrm{Yu}$ SH provided insights for the study. Zhu Y supervised this study and revised the manuscript. All authors contributed to the general discussion and commented on the manuscript.

Conflict of interest The authors declare no competing financial interest.

Supplementary information Supporting data are available in the online version of the paper. 


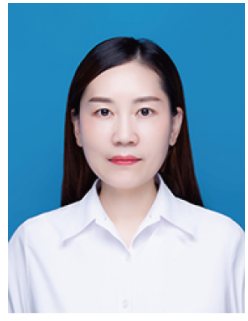

Hong Yuan received her bachelor's degree in material chemistry from Liao Cheng University in 2014. She completed her master's degree in materials engineering from Lanzhou Institute of Chemical Physics, Chinese Academy of Sciences in 2017. She is currently working toward the $\mathrm{PhD}$ degree in material physics and chemistry at the University of Science and Technology of China and her current research focuses on the interfacial construction and thermal mechanical properties of graphene assembly.

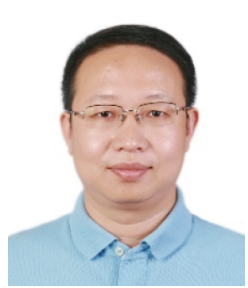

Yanwu Zhu is currently a professor at the Department of Materials Science and Engineering, University of Science and Technology of China. He received his MSc (2003) from Peking University and $\mathrm{PhD}$ (2007) from the National University of Singapore (NUS), both in physics. He was a postdoctoral researcher at the NUS and the University of Texas at Austin. His research focuses on the preparation, characterization and properties of graphene and other novel carbon materials.

\section{基于 $\pi-\pi / \mathrm{H}$-键协同作用制备含芘小分子增强高韧} 性石墨烯纸

袁宏 ${ }^{1}$, 葛良兵 ${ }^{1}$, 倪驾 ${ }^{1}$, 阙秀凯 ${ }^{3}$, 陈思铭 ${ }^{2}$, 高梦婷 ${ }^{3}$, 潘飞 ${ }^{1}$, 叶江林 ${ }^{1}$, 许方 ${ }^{1}$, 束娜 ${ }^{1}$, 李婕云 ${ }^{1}$, 索涛 ${ }^{3}$, 俞书宏 ${ }^{2}$, 朱彦武 ${ }^{1 *}$

摘要 轻质高强且具备高韧性的石墨烯组装材料在抗冲击防护领 域有着潜在的应用价值. 在这项工作中, 我们通过在还原氧化石墨 烯层间界面交联1-氨基芘 $\mathrm{AP}$ 和 1 - 芘丁酸 $\mathrm{PB}$ 共轭小分子, 可以获得 具有高导电、高韧性的超强复合石墨烯纸(AP/PB-GPs). 结果表明, 超过 $10 \mu \mathrm{m}$ 厚度的复合石墨烯纸具有超高的平均韧性( 69.67 \pm $15.3 \mathrm{MJ} \mathrm{m}^{-3}$ ), 同时抗拉伸强度接近 $1 \mathrm{GPa}$; 尤其在抗冲击性能方 面, 在高速弹道冲击速度下, 仍然可以获得优异的比穿透能量吸收 值 $\left(0.17 \mathrm{MJ} \mathrm{kg} \mathrm{kg}^{-1}\right)$. 详细的界面和结构分析表明, 界面增强是由相 邻石墨烯层间与共轭分子之间的 $\pi-\pi$ 相互作用和氢键连接共同决 定的. 尤其是石墨烯纳米片内的孔洞及边缘缺陷更有利于共轭小 分子充分的吸附, 这必然会使界面结合最大化, 在连续高的加载应 力下能够有效促进裂纹的偏转和塑性变形. 密度泛函理论(DFT)模 拟表明, 石墨烯纳米片边缘的-COOH极性官能团与 $\mathrm{AP} / \mathrm{PB}$ 分子表 面的 $-\mathrm{NH}_{2} 、-\mathrm{COOH}$ 之间的耦合对氢键网络的形成起着关键作用. 